\section{Fokale Laserablation bei Prostatakarzinom}

Natarajan S Et Al. Focal Laser Ablation Of Prostate Cancer: Phase I clinical trial. J Urol 2016; 196: 68-75

Die fokale Laserablation stellt eine neue Möglichkeit zu Behandlung von umgrenzten Prostatakarzinomen dar. Dabei wird lediglich der mittels multiparametrischer MRT identifizierte Tumor plus Sicherheitsabstand mit dem Laser koaguliert, sodass die behandlungsbedingten Komplikationen im Vergleich zu ausgedehnteren Verfahren geringer sein sollen. Kalifornische Mediziner haben die Durchführbarkeit und Sicherheit der Methode untersucht.

Natarajan et al. haben 8 Männer mit einem singulären Prostatakarzinomherd Stadium $\leq \mathrm{T} 2 \mathrm{~b}$ und Gleason-Score $\leq 7(3+4)$ in die Phase-I-Studie aufgenommen. Die Laserfasern wurden in Lokalanästhesie unter MRT-Kontrolle in der Prostata platziert. Zusätzlich führten transrektal die Wissenschaftler transperineal 2 oder 3 Temperatursonden in die Prostata ein, mindestens eine davon in unmittelbarer Nähe zum Rektum. Die Laserbehandlung erfolgte mit einer Leistung von 11 bis $14 \mathrm{~W}$ mit im Mittel 7 Applikationen pro Patient. Die gesamte Dauer des Eingriffs einschließlich Vorbereitung betrug durchschnittlich knapp 5 Stunden.

Als primären Endpunkt beurteilten die Mediziner die Komplikationsrate. Weitere exploratorische Endpunkte umfassten Veränderungen im International Prostate Symptom Score (IPSS) und International Index of Erectile Function (IIEF) sowie den histologischen Nachweis von Tumorrestgewebe und -rezidiven in der Biopsie 6 Monate nach dem Eingriff. Die Auswertung ergab
- insgesamt 23 unerwünschte Ereignisse vom Schweregrad 1 oder 2 über 9 Monate postoperativ,

- keine Ereignisse vom Schweregrad 3 oder höher und

- keine Fälle von Harninkontinenz und erektilen Funktionsstörungen laut IPSS und IEEF.

Die mittlere Temperatur außerhalb des Ablationsfelds lag in allen Fällen unter $40^{\circ} \mathrm{C}$, wobei die MRT-Thermometrie anfällig gegenüber Bewegungsartefakten war. Bei 6 der 8 Männer lieferten die Temperatursonden dagegen auswertbare Daten. Das durchschnittliche Prostatavolumen nahm nach 6 Monaten von präoperativ $35,5 \mathrm{~cm}^{3}$ auf $32,5 \mathrm{~cm}^{3}$ ab. In der zu diesem Zeitpunkt durchgeführten Biopsie fanden sich bei 5 der 8 Männer im behandelten Bereich keine Tumorreste. In den benachbarten Zonen waren allerdings bei 6 Männern persistierende Tumoren nachweisbar, 3 davon mit eine Gleason-Score von $7(3+4)$ bzw. 8. Ein weiterer Patient zeigte ein Karzinom in weiter Entfernung von der Ablationszone, und ein Mann war vollständig tumorfrei.

\section{FAZIT}

Die Laserablation bei lokal begrenztem Prostatakarzinom mit insgesamt mäßigem Risiko scheint ohne schwere Komplikationen durchführbar, so die Autoren. Als Vorteil sehen sie auch, dass keine Allgemeinnarkose notwendig ist und die Patienten nach wenigen Stunden aus der Klinik entlassen werden können. Die onkologischen Ergebnisse scheinen dagegen weniger zufriedenstellend - möglicherweise muss der Sicherheitsabstand um den sichtbaren Tumor herum über die hier verwendeten $10 \mathrm{~mm}$ erweitert werden.

\section{Dr. Elke Ruchalla, Bad Dürrheim}

\section{Kommentar}

Im Gegensatz zur roboterassistierten radikaIen Prostatektomie mit dem weltweit etablierten DaVinci ${ }^{\circledR}$-System in der Monopolstellung drängen in der fokalen Therapie des Prostatakarzinoms zahlreiche Systeme auf den Markt. Die Nische zwischen aktiver Überwa- 
chung und Ganzdrüsentherapie soll besetzt werden. Das fordern auch die Patienten, die z.T. aktiv nach fokalen Therapiealternativen suchen. Deshalb gewinnt das Wissen um die verschiedenen Systeme für die Urologie an Bedeutung.

Diese Studie untersuchte die „focal laser ablation“ oder „laser interstitial thermal therapy (LITT) “ genannt. Die LITT wird in der Neurochirurgie u. a. genutzt um MR-gesteuert kleinste Hirnareale bei Epilepsie- und TumorPatienten zu zerstören. Dies wird ermöglicht durch die hier ebenfalls genutzten MRT-kompatiblen Laserfasern, die in Kühlkathetern platziert werden, um hierdurch zielgenau Energie zu applizieren. In der Urologie ist dieser Laser für die Behandlung der BPO durch optimierte Laser-Systeme vom Markt verdrängt worden, die vermutlich zu einer geringeren Inkontinenz führen [1]. Arbeiten aus unserer Klinik zeigten für diesen 980 nm Diodenlaser eine Koagulationstiefe bei $10 \mathrm{~W}$ von ca. $0,9 \mathrm{~mm}$ (Nutzung für die fokale Therapie in der Studie 12-15W) [2].

Den Autoren gebührt Anerkennung für die Durchführung dieser aufwendigen interdisziplinären Behandlung im MRT. Ein Abstand zum Sphinkter externus Vesicae von mind. $2 \mathrm{~cm}$ führte in der Studie sicherlich zu den wenigen Komplikationen, schränkt möglicherweise die zukünftige Nutzung ein. Das Karzinom war bei wenigen Patienten nach 6 Monaten histologisch nicht mehr nachweisbar. Ist dies nur eine Frage des zu geringen Sicherheitsabstandes? Die Lasersonden wurden stereotaktisch platziert und die Lage im MRT überprüft. Möglicherweise verhindert die zur Identifikation der Laserfaser in der Prostata notwendige Testdosis eine genaue Replatzierung und Behandlung. Hier scheint Forschungsbedarf ex vivo zu bestehen. $2 \mathrm{~Pa}$ tienten hatten mit PSA-Werten $>15 \mathrm{ng} / \mathrm{ml}$ Tumorcharakteristika, die in keinen Consensus-Empfehlungen als geeignete Patienten für die fokale Therapie empfohlen werden. Dies deutet auf die schwierige Patientenselektion für die fokale Therapie hin. Eine zeitgleich publizierte Studie von Lepor et al. zur LITT zeigt gegensätzliche Daten [3]. $25 \mathrm{~Pa}$ tienten mit ähnlichen Einschlusskriterien zeigten nach 3 Monaten eine Tumorfreiheitsrate von $96 \%$. Auf Basis dieser gegensätzlichen onkologischen Ergebnisse und wenig behandelten Patienten ist eine Gesamteinschätzung des Potentials der LITT nicht mög- lich. Studien mit größeren Patientenkohorten sind notwendig.

Als Fazit für die Praxis kann geschlossen werden, dass die LITT weit entfernt ist vom Routineeinsatz. Patienten mit unifokalen niedrigfrüh intermediären Tumoren, die eine fokale Therapie wünschen, müssen über den experimentellen Charakter aufgeklärt werden und sollten ausschließlich in Studien behandelt werden.

Der Autor

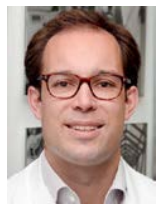

Dr. Jost von Hardenberg Klinik für Urologie, Universitätsmedizin Mannheim, Universität Heidelberg jost.vonhardenberg@medma. uni-heidelberg.de

\section{Literatur}

[1] Herrmann TR, Liatsikos EN, Nagele U et al. EAU Guidelines on Laser Technologies. Eur Urol 2012; 61: 783-795

[2] Wezel F, Wendt-Nordahl G, Huck N et al. New alternatives for laser vaporization of the prostate: Experimental evaluation of a 980-, 1,318- and 1,470-nm diode laser device. World J Urol 2010; 28: 181-186

[3] Lepor H, Llukani E, Sperling D et al. Complications, recovery, and early functional outcomes and oncologic control following in-bore focal laser ablation of prostate cancer. Eur Urol 2015; 68: 924-926 